\title{
FUSION OF HYPERSPECTRAL AND MULTISPECTRAL IMAGERY WITH REGRESSION KRIGING AND THE LULU OPERATORS; A COMPARISON
}

\author{
Nithya Jeevanand ${ }^{1, *}$, Mr Prabhakar Alok Verma ${ }^{1}$, Dr.Sameer Saran ${ }^{1}$ \\ ${ }^{1}$ Geoinformatics Department, Indian Institute of Remote Sensing, Dehradun, India - nithya25594@gmail.com, \\ (prabhakar, Sameer)@iirs.gov.in
}

Commission V, SS: Emerging Trends in Geoinformatics

KEY WORDS: Image fusion, Regression Kriging, LULU operators, Classification, Slums

\begin{abstract}
:
In this digital world, there is a large requirement of high resolution satellite image. Images at a low resolution may contain relevant information that has to be integrated with the high resolution image to obtain the required information. This is being fulfilled by image fusion. Image fusion is merging of different resolution images into a single image. The output image contains more information, as the information is integrated from both the images Image fusion was conducted with two different algorithms: regression kriging and the LULU operators. First, regression Kriging estimates the value of a dependent variable at unsampled location with the help of auxiliary variables. Here we used regression Kriging with the Hyperion image band as the response variables and the LISS III image bands are the explanatory variables. The fused image thus has the spectral variables from Hyperion image and the spatial variables from the LISS III image. Second, the LULU operator is an image processing methods that can be used as well in image fusion technique. Here we explored to fuse the Hyperion and LISS III image. The LULU operators work in three stages of the process, viz the decomposition stage, the fusion and the reconstruction stage. Quality aspects of the fused image for both techniques have been compared for spectral quality (correlation) and spatial quality (entropy). The study concludes that the quality of the fused image obtained with regression kriging is better than that obtained with the LULU operator.
\end{abstract}

\section{INTRODUCTION}

Land use describes the way the land is used and the resources are managed and maintained. Land use is a topic of a wide interest and has been studied extensively. It contains various human activities like transport, agriculture, settlements, and parks. Land use is categorized commonly into urban and rural land use, the distinction being made on the basis of population density (M. Li, Stein, Bijker, \& Zhan, 2016). Urban land use, in turn, is categorized into residential land use, commercial land use, transportation-land use, industrial land use, open space and recreational land use. Rural land use is land for both agriculture and residential use. "Remote sensing is defined as the science and technology by which characteristics of objects of interest can be identified without direct contact" (Gunda, 2014). It helps to observe size, shape, and character of land use and land cover without direct contact by taking satellite images. Fusion is defined as combining two different entities into a single entity. Here we consider image fusion. Image fusion is defined as the process of integrating two or more different images into a single image (Pohl \& van Genderen, 1998). Additional benefits of image fusion are that it may reduce the computational cost and that it is less time consuming while performing with the algorithms. It is also useful in different fields of application like defense (Ojeda, Britos, \& Vallejos, 2017), population count (Jain, 2007). Image fusion as used in this research aims to reduce the number of artifacts, being errors made by humans during processing or instrument errors. The instrument error has been experienced in the dataset of Hyperion image that contains noise which is been created by the sensors of the

\footnotetext{
${ }^{*}$ Corresponding author
}

earth observing satellite. The main aim of this research is to perform image fusion for identifying the informal settlements in Rishikesh City. The scope of this research is to identify the informal settlements and provide directions on how to supply the necessary aids to the people located in the slum and to help the ministry of urban and poverty alleviation department to achieve their aim of slum free India. Although eradication of slum is not possible, people in the informal settlement have the right to acquire all the benefits from the society. In order to achieve the aim of this research, we have as the main objective of this research to perform image fusion with the low resolution and high-resolution satellite imagery.

\section{STUDY AREA AND DATASETS}

\subsection{Study Area}

The study area for this research is Rishikesh, Uttarakhand, India. The area is extends from $30^{\circ} 0^{\prime} 30^{\prime \prime} \mathrm{N}$ to $78^{0} 14^{\prime} 50^{\prime \prime} \mathrm{E}$ and $30^{\circ} 6^{\prime} 30^{\prime \prime} \mathrm{N}$ to $78^{\circ} 11^{\prime} 30^{\prime \prime} \mathrm{E}$. The area contains a glass factory which manufactures glass and enrols people from different regions of the country. Mostly, people who migrate from one place to another involve in building the informal settlements. The migrated people from different regions come in search of jobs. The informal settlements are not registered by the government, so those settlements are illegal settlements. The housing of the settlements are irregular, the roof tops are covered with plastic sheets with no ventilation and no proper sanitation. The people live with their families and they have to undergo many troubles, refer appendix to visualize the slum area in Rishikesh City. The hyperspectral and the multispectral image of the same year were available. The field survey was conducted in February 2018. 


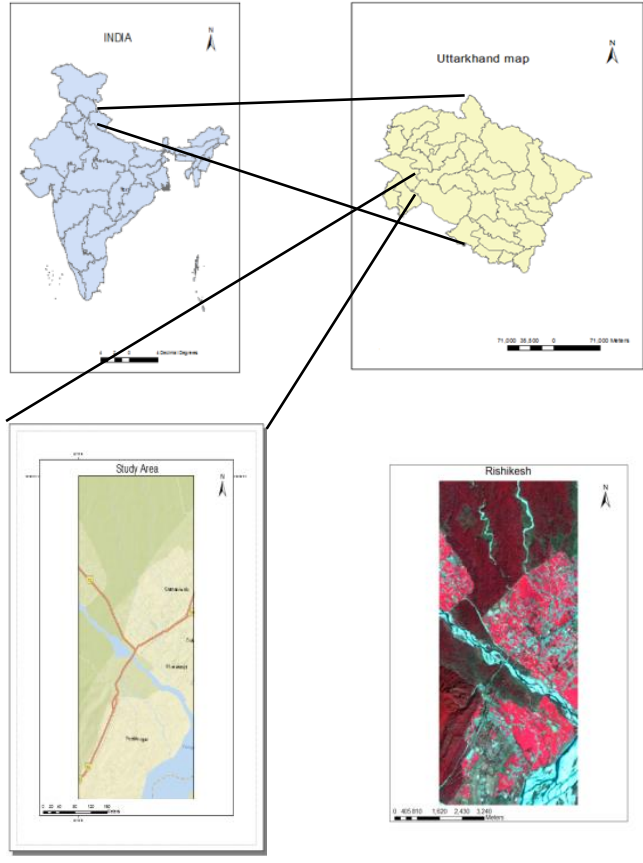

Figure 1 : Study Area

\subsection{Dataset}

The aim is to explore the presence of slum dwellings from remote sensing images. The reason to do so is that the slums are changing rapidly and remote sensing images are useful to perform a standardized monitoring of the development. In this research, Hyperion is hyperspectral imagery, as Earth-observing Satellite launched on $21^{\text {st }}$ November 2000.The spectral classes of vegetation, water can been seen but spatially we cannot view it. LISS III (Linear Imaging Self Scanning Sensor) is a multispectral imagery, IRS-1C (Indian Remote Sensing) was launched on $28^{\text {th }}$ December 1995, spectrally of the image is less compared to hyperspectral image. These images are used for fusion to obtain the spectral values of Hyperion and spatial values of LISS-III. The hyperion has pre-processing steps due to the error in the camera calibration.

\section{Table 1: Dataset Table}

\begin{tabular}{|l|l|l|}
\hline Specification & Hyperion & LISS-III \\
\hline $\begin{array}{l}\text { Spatial Resolution } \\
(\mathrm{m})\end{array}$ & $30 \mathrm{~m}$ & $23.5 \mathrm{~m}$ \\
\hline Spectral bands & VNIR (70 & $0.52 \mu \mathrm{m}-$ \\
& channels, & $0.59 \mu \mathrm{m}$ \\
& $356 \mathrm{~nm}-$ & $0.62 \mu \mathrm{m}-$ \\
& $1058 \mathrm{~nm})$, SWIR & $0.68 \mu \mathrm{m}$ \\
& $(172 \mathrm{channels}$, & $0.77 \mu \mathrm{m}-0.86$ \\
& $852 \mathrm{~nm}-$ & $\mu \mathrm{m}$ \\
& $2577 \mathrm{~nm})$ & $1.55 \mu \mathrm{m}-$ \\
& & $1.70 \mu \mathrm{m}$ \\
\hline Swath Width $(\mathrm{km})$ & 35 & 141 \\
\hline
\end{tabular}

\section{METHODOLOGY}

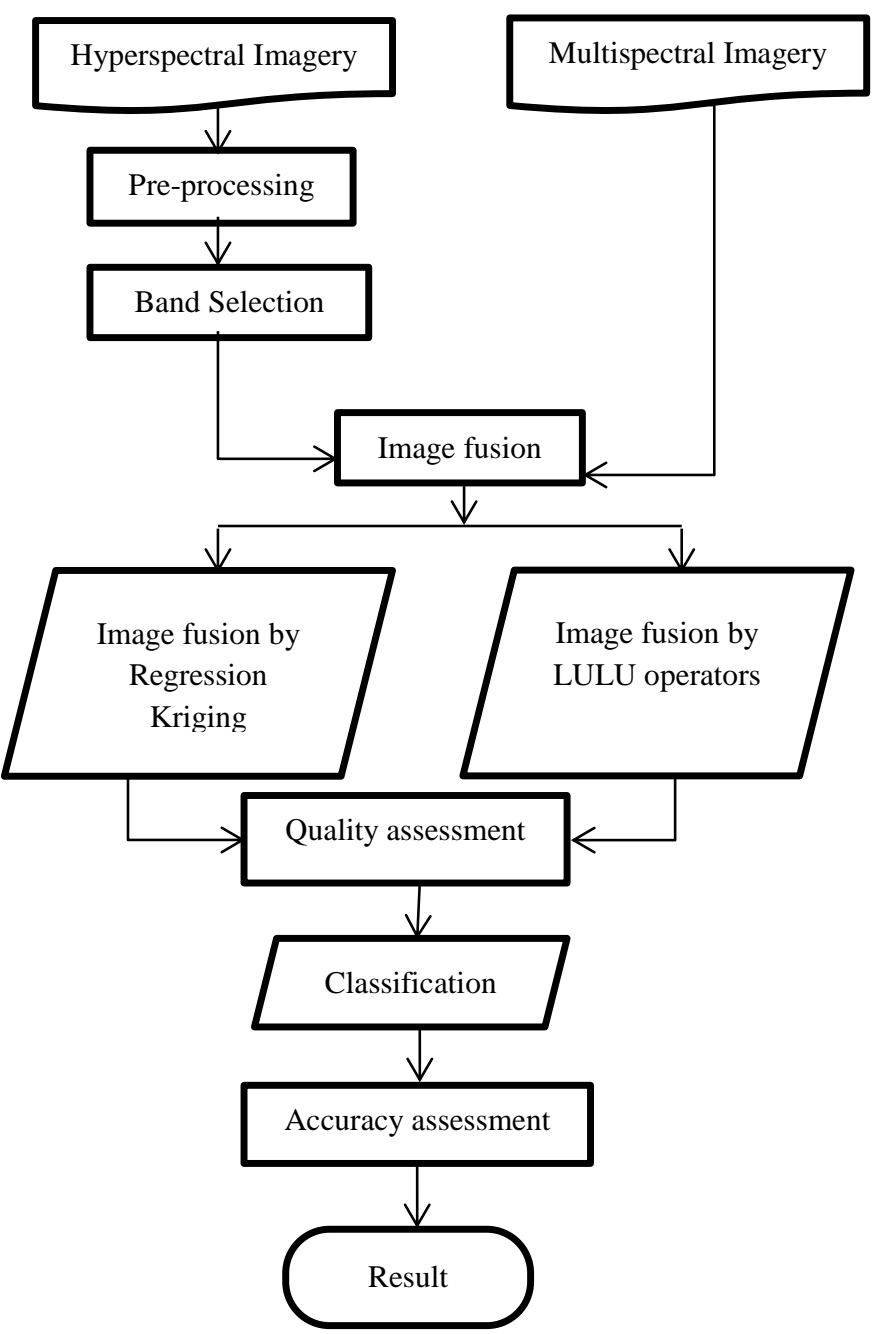

Figure 2: Methodology

\section{Regression Kriging}

Regression kriging is a spatial interpolation method that helps in estimating the values of a variable at an unvisited location by applying regression on the dependent variables and auxiliary variables, followed by simple kriging of the residuals (Hengl et al., 2007). Simple kriging can be used as the residuals have a mean equal to zero.

$$
\hat{\mathrm{x}}\left(\mathrm{s}_{0}\right)=\sum_{\mathrm{k}=0}^{\mathrm{p}} \widehat{\beta_{\mathrm{k}}} \mathrm{q}_{\mathrm{k}}\left(\mathrm{s}_{0}\right)+\sum_{\mathrm{i}=1}^{\mathrm{n}} \lambda_{\mathrm{i}} \widehat{\mathrm{z}_{1}}\left(\mathrm{~s}_{\mathrm{i}}\right)
$$

$\widehat{\beta_{k}}=$ The estimated regression coefficient

$q_{k}\left(\mathrm{~s}_{0}\right)=$ auxiliary variables of the target location $\lambda_{i}=$ kriging weights

$\hat{Z}\left(s_{i}\right)=$ residuals at location $s_{i}$ (Hengl et al., 2007).

\section{LULU Operators}

We consider an image consisting of pixels $V$. The general formula of LULU operator equals

$$
L_{n}(f)(x)=\max _{V \in N_{n}(x)} \min _{y \in V} f(y), \quad x \in z^{2} \quad n=
$$

$$
1,2,3, \ldots
$$


$U_{n}(f)(x)=\min _{V \in N_{n}(x)} \max _{y \in V} f(y), x \in z^{2} \quad n=$

$1,2,3, \ldots$.

where

$V \in N_{n}(x)=$ a neighbourhood pixels of $x$

$N_{n}(x)=$ set of all connected sets of size $n$ around the pixel $x$.

$z^{2}=$ domain of the image $f(x)$, reflecting a twodimensional image

$L_{n}(f)(x)$ and $U_{n}(f)(x)$ are the lower and upper operator operates on image $f$ with a function of the pixel. For each pixel $x$, we consider the image $f(x)$ and we take either the upper operator $U$ of size $n$ or the lower operator $L$ of size $n$. In order to do so, we use the neighbourhood of size $n$ around $x$, namely $N_{n}(x)$. For $L_{n}(f)(x)$ we take the maximum overall the sets $V$ that are neighbourhood of size $n$ of the pixel $x$, followed by minimizing overall the pixel in $V$. For $U_{n}(f)(x)$ we take the minimum overall the sets of $V$ that are neighbourhood of size $n$ of the pixel $x$, followed by maximizing overall the pixels in $V$.

$$
\begin{aligned}
& \operatorname{supp}\left(\varphi_{n_{1} s_{1}}^{*}\right) \cap \operatorname{supp}\left(\varphi_{n_{2} s_{2}}^{*}\right) \neq \emptyset \Rightarrow \\
& \operatorname{supp}\left(\varphi_{n_{1} s_{1}}^{*}\right) \subset \operatorname{supp}\left(\varphi_{n_{2} s_{2}}^{*}\right)
\end{aligned}
$$

The above equation shows the decomposition process with minimum and maximum sets or disjoints sets here $*$ is represented to show the decomposition. This equation plays a role in the pre-merging steps which is mentioned below where new interval is being created. Image fusion is basically about reconstruction. Reconstruction is performed by adding layers, where layers are the decomposed sets of images. A partial reconstruction is performed by creating subset layers and creating a set of subsets for each layer: partial reconstruction plays an important role for fusion. This is being done by creating subset layers and for each layer a set of subsets is created. If data compression is taken into account then the coefficients of wavelets are commonly used, wavelets are orthonormal series of square integrable functions. The symbol of wavelet is $\psi$, the coefficient of wavelet is defined as $c_{j k}$ equals

$$
c_{j k}=\left[W_{\psi} f\right]\left(2^{-j}, k 2^{-j}\right)
$$

where $a=2^{-j}$ refers to the (binary) dilation and $b=k 2^{-j}$ to the (binary or dyadic) position.

Quantization is a compression technique that compresses a range of values into a single value. The non-zero quantized value is stored or transmitted. The local frequency equals the squares of the coefficient values of the wavelets and support function at the chosen location (I. Fabris-Rotelli \& Van Der Walt, 2009). LULU fusion is defined as the application of the discrete pulse transform in the pixels of the input images. The image undergoes decomposition into individual pixels; there are different procedures available for obtaining the connectivity trees. The connectivity trees are hierarchical tree created and labelled, so that it is fused with the help of the newly created discrete pulse with the help of sort and join method. In this study, we created the connectivity trees with the help of the union-find algorithm from the pixel of the images (I. Fabris-Rotelli \& Van Der Walt, 2009). This algorithm labels each tree with a unique label. The new pulse helps the decomposed image to fuse because the pulse is subject to the Digital Pulse Transform fusing the information (spectral information as well as spatial information) present in the images. The fused image is retained from the partial reconstruction of an image.

\section{RESULTS}

\section{Pre-processing of Hyperion Bands}

The hyperion is of 242 bands which is not atmospherically corrected for that the pre-processing steps is conducted.

\section{Bad Band Removal}

The hyperspectral image is taken as Hyperion which is not atmospherically corrected. To perform atmospheric correction we process the image by removing noise, empty bands. 103 bands are obtained from 242 bands which is used for further process.

\section{Along-track destriping}

The band that contains a vertical strip of black, white or sometimes both color strips which has no information in it. Thus, the uninformative pixel location will be both spatially and spectrally of the given area that specifies 0 . The values can be removed by the averaging the neighboring values (Han, Goodenough, Dyk, \& Love, 2002). For all the 103 bands the both color strips are removed.

\section{Atmospheric correction}

Atmospheric correction is applied to improve the quality of the image; the properties are studied for the removal of atmospheric absorption and scattering effects. The parameters of the atmospheric correction are aerosol distribution, visibility, water vapor (Bhatt, 2017). FLAASH (Fast line of sight) retrieves spectral reflectance from hyperspectral radiance images.

\section{Band Selection}

The resultant image undergoes image to image registration (with Landsat 8) and to reduce the computational error, the bands are selected. The band selection is performed with the help of correlation method. The correlation value is compared; the highest correlation value is taken into consideration. As a result 45 bands are selected for further process.

\section{Regression kriging}

The fusion is performed with the Hyperion image and LISS III image, from the figure 2 we can say that the spatial resolution of Hyperion is high compared to the spatial resolution of LISS III image. According to image fusion, the spatial resolution has to be taken from the LISS III Image because it has a high spatial resolution which is helpful in different fields of application. The spatial location is taken from the LISS III image and the pixel values are taken from the 
Hyperion image. The spectral resolution is obtained from the Hyperion image, as Hyperion image contains a high spectral resolution. In other words, the fused output contains high spectral resolution and high spatial resolution. The output fused image appears to smoothen but the information present in the image is preserved.

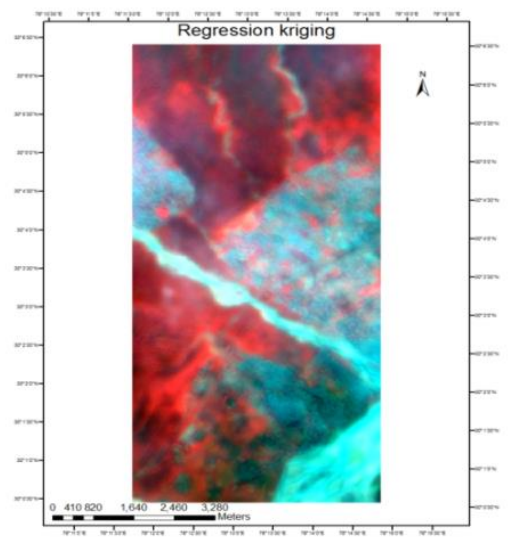

Figure 3: Fused Image of Regression Kriging

\section{LULU operators}

The spectral and spatial values are fused properly with the LISS III image is been interpreted. The output of decomposition stage was in the form of the dictionary where each and every variable has a new address. With the help of the values in each dictionary, the boundary position and the number of non-zeroes present in the variable is found. The fusion occurs with the values of boundary position present for each variable with a comparison to the total area by which the spectral values, as well as the spatial values, are fused in an apparent way. The reconstruction stage of the image appears to be in the matrix form after undergoing the hierarchical tree structure.

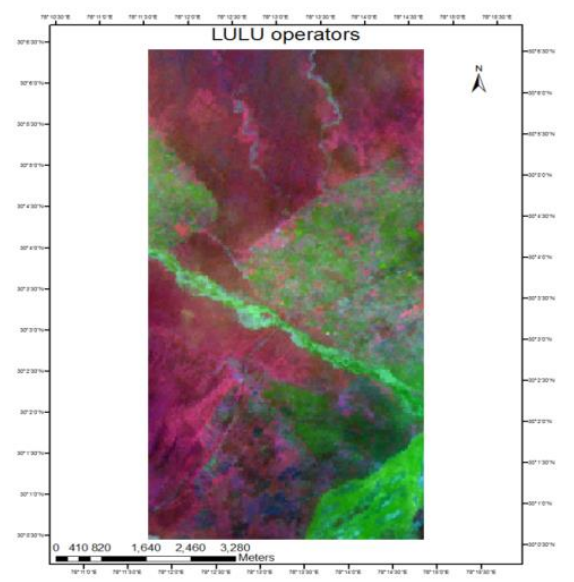

Figure 4: Fused Image of LULU operators

\section{Quality Comparison}

\section{Entropy}

The quality comparison is carried out to know, the amount of information preserved by both the methods with a comparison to Hyperion image. Entropy calculated to find out the improvement in the spatial resolution of Hyperion image. The table 1 represents the amount of information present in every band with a comparison to the reference image. The average entropy of reference image is 10.884 . The average entropy of fused image of regression kriging is 12.553 and the average of fused image of LULU operators is 11.360 .

Table 2: Overall Entropy

\begin{tabular}{|l|l|}
\hline $\begin{array}{l}\text { Hyperion (Reference } \\
\text { Image) }\end{array}$ & Average Entropy \\
\hline $\begin{array}{l}\text { Fused image by } \\
\text { Regression Kriging }\end{array}$ & 12.552885 \\
\hline $\begin{array}{l}\text { Fused image by } \\
\text { LULU operators }\end{array}$ & 11.360132 \\
\hline
\end{tabular}

\section{Correlation}

The spectral values present in the fused output of regression kriging and LULU operators by comparing with the original input image. The correlation is calculated between Reference image and the fused image of both techniques and the overall correlation of reference image with Regression kriging fused image is 0.87718 and for LULU operators is 0.98040 . Thus, the LULU operators have preserved more spectral values compared to regression kriging method.

Table 3: Overall Correlation

\begin{tabular}{|l|l|}
\hline & $\begin{array}{l}\text { Overall } \\
\text { Correlation }\end{array}$ \\
\hline $\begin{array}{l}\text { Correlation between } \\
\text { Hyperion and Fused } \\
\text { image of Regression } \\
\text { Kriging }\end{array}$ & 0.87718 \\
\hline $\begin{array}{l}\text { Correlation between } \\
\text { Hyperion and Fused } \\
\text { image of LULU } \\
\text { Operator }\end{array}$ & 0.98040 \\
\hline
\end{tabular}

\section{Classification}

The classification process is carried out in maximum likelihood classifier. To perform the maximum likelihood classifier, the region of interest is created. For example, the class forest which is represented in red color is created by creating $n$ number of classes of a same spectral feature or same region of interest. By this six spectral classes are created. Then the fused output image by regression kriging method is classified by using maximum likelihood classifier for detecting the informal settlements. 


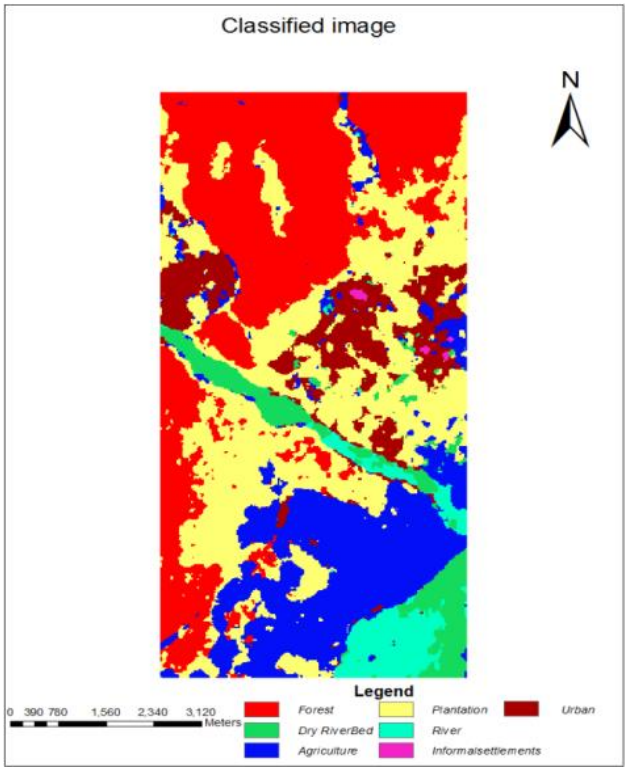

Figure 5: Classified Image

\section{Accuracy Assessment}

After obtaining the classified output, the accurate position of the informal settlements has to be estimated. The accuracy is estimated by creating a new region of interest and the newly created points are overlaid on the classified image

Table 4: Accuracy assessment

\begin{tabular}{|l|l|}
\hline Overall accuracy & 0.73488 \\
\hline$\kappa$ coefficient & 0.6611 \\
\hline User's Accuracy & 1 \\
\hline Producer's Accuracy & 0.09824 \\
\hline Errors of Commission & 0 \\
\hline Errors of Omission & 0.90176 \\
\hline
\end{tabular}

\section{CONCLUSION}

In the field of remote sensing we require high resolution images, the images are obtained by image fusion. There are many existing algorithms for image fusion. Thus, we have obtained the image by using regression kriging and LULU operators, thus the informal settlements are identified by using maximum likelihood classifier. Further work can be using different satellite image and for other fields of application also.

\section{REFERENCES}

Anguelov, R., \& Fabris-Rotelli, I. (2010). LULU Operators and Discrete Pulse Transform for Multidimensional Arrays. IEEE Transactions on Image Processing, 19(11), 3012-3023.

Anguelov, R., \& Plaskitt, I. (2007). A Class of LULU Operators on Multi-Dimensional Arrays. Computer and Pattern Recoginition, 1-19.
Bhatt, H. (2017). Comparison of AVIRIS - NG with EO - 1 Hyperion Data for Mineral Identification. In acrs (pp. 1-37). Delhi.

Boudraa, A.-O., Bentabet, A., Salzenstein, F., \& Guillon, L. (2004). Dempster-Shafer 's Basic Probability Assignment Based on Fuzzy Membership Functions. Electronic Letters on Computer Vision and Image Analysis, 4(1), 1-9.

Dutta, P. (2017). An Uncertainty Measure and Fusion rule for Conflict Evidences of Big Data Via DempsterShafer Theory. International Journal of Image and Data Fusion, $\quad 00(00), \quad 1-18$. https://doi.org/10.1080/19479832.2017.1391336

Fabris-Rotelli, I. (2011). The Discrete Pulse Transform for Images with Entropy-Based Feature Detection. In PRASA Processing. Pretoria, South Africa.

Fabris-Rotelli, I., \& Van Der Walt, S. J. (2009). The Discrete Pulse Transform in Two Dimensions. In Proceedings of the twentieth annual symposium of the Pattern Recognition Association of South Africa (PRASA).

Hengl, T., Heuvelink, G. B. M., \& Rossiter, D. G. (2007). About Regression-Kriging: From Equations to Case Studies. Computers and Geosciences, 33(10), 1301-1315. https://doi.org/10.1016/j.cageo.2007.05.001

Li, M., Stein, A., Bijker, W., \& Zhan, Q. (2016). Urban Land Use Extraction From Very High Resolution Remote Sensing Images by Bayesian Network. ISJournal of Photogrammetry and Remote Sensing, 122, 192-20

Jain, S. (2007). Use of IKONOS Satellite Data to Identify Informal Settlements in Dehradun, India. International Journal of Remote Sensing, 28(15), 32273233. https://doi.org/10.1080/01431160600705122

Ojeda, S. M., Britos, G. M., \& Vallejos, R. (2017). An Image Quality Index based on Coefficients of Spatial Association with an Application to Image Fusion. Spatial Statistics, 23, 1-16.

Pajares, G., \& de la Cruz, J. M. (2004). A Wavelet-based Image Fusion Tutorial. Pattern Recognition, 37(9), 1855-1872. https://doi.org/10.1016/j.patcog.2004.03.010

Pardo-Igzquiza, E., Chica-Olmo, M., \& Atkinson, P. M. (2006). Downscaling Cokriging for Image Sharpening. Remote Sensing of Environment, 102(1-2), 86-98.

Pohl, C., \& van Genderen, J. . (1998). Review article Multisensor image Fusion in Remote Sensing: Concepts, Methods and Applications. International Journal of Remote Sensing, 19(5), 823-854.

Richards, J. a. (2006). Remote Sensing Digital Image Analysis. Springer Berlin Heidelberg.

Rohwer, C. (2005). Nonlinear Smoothing Multiresolution. (K.-H. H. Bonn \& D. M. Tempe, Eds.). Berlin: Springer Berlin Heidelberg. 
Stein, A., \& Corsten, L. C. A. (1991). Universal Kriging and Cokriging as a Regression Procedure. Biometric, $47(2), 575-587$.

Yakhdani, M. F., \& Azizi, A. (2010). Quality Assessment of Image Fusion Techniques for Multisensor High Resolution Satellite Images (Case study: IRS-P5 AND IRS-P6 satellite images). International Archives of the Photogrammetry, Remote Sensing and Spatial Information Sciences - ISPRS Archives, 38, 204-209. 